diagnosis of PSC. We audited the colitis surveillance against audit standards published in the BSG and UK PSC guidelines. Methods All UK PSC investigators were invited (March 2019Jan 2021) to complete an electronic questionnaire encompassing demographics, diagnosis and bowel cancer surveillance data on each patient with PSC under the care of their service. Results 1,795 patients across 30 centres (liver units $n=1548$, general gastroenterology units $n=247$ ) were included. Median age at diagnosis was 51 years and $56.4 \%$ were men.

Concurrent IBD was present in 1264 patients (70.4\%) with $256(20.3 \%)$ having had a colectomy. Where classified, colitis was present in 924/939 (98.4\%) patients whereas isolated ileal disease was present in 15/939 patients. Pancolitis (Montreal classification E3) was the commonest disease distribution (673/ 939, 71.7\%).

Most patients with IBD were followed up by an IBD specialist $(n=616,48.7 \%), 266(21.1 \%)$ were followed by a general gastroenterologist, $236(18.7 \%)$ by a hepatologist, whereas $15(1.2 \%)$ patients were followed in a joint IBD/Hepatology clinic.

Among those with colitis without previous colectomy $(n=743), 580(78.1 \%)$ underwent annual colonoscopic surveillance; 30 (5.2\%) with dye spray, 230 (39.7\%) with biopsies and dye spray, and $252(43.4 \%)$ with protocol biopsies alone.

Of those without documented IBD diagnosis, only 303/507 $(59.7 \%)$ had this excluded by colonoscopy and biopsies.

Age $<40$ was associated with poorer compliance with colonoscopy surveillance. $(\mathrm{P}=0.023)$.

Conclusions IBD screening and colonic cancer surveillance is suboptimal in this large UK cohort of patients with PSC. This highlights the need for awareness of PSC-IBD management to address this unwarranted variation in care of people with PSC in the UK.

\section{PM0-36 SAFETY AND EFFICACY OF USTEKINUMAB FOR CROHN'S DISEASE IN THE ELDERLY POPULATION}

${ }^{1}$ Joseph Fiske*, ${ }^{2}$ Eleanor Liu, ${ }^{2}$ Jimmy Limdi, ${ }^{1}$ Thomas Conley, ${ }^{1}$ Tristan Townsend, ${ }^{3}$ Michael Davies, ${ }^{3}$ Robert Brockwell, ${ }^{1}$ Daniyal Baig, ${ }^{4}$ Angela Liaros, ${ }^{1}$ Sreedhar Subramanian. ${ }^{1}$ Liverpool Foundation Trust, Liverpool, UK; ${ }^{2}$ Pennine Acute Hospital NHS Trust, Bury; ${ }^{3}$ Arrowe Park Hospital, Wirral, UK; ${ }^{4}$ Whiston Hospital, Whiston, UK

\subsection{6/gutjnl-2021-BSG.175}

Introduction The rising incidence of inflammatory bowel disease (IBD) worldwide and an ageing population has led to a marked increase in elderly IBD patients. Anti-tumour necrosis factor (TNF) agents are associated with an increased risk of serious infections and treatment discontinuation among elderly IBD patients; little is known about non anti-TNF biologics in this cohort. We aimed to examine safety and efficacy of ustekinumab in elderly Crohn's disease (CD) patients.

Methods Patients $\geq 60$ years old commencing ustekinumab for $\mathrm{CD}$ were included in this retrospective multi-centre cohort study. We gathered data on adverse events, Harvey Bradshaw Index (HBI) and steroid therapy. The primary outcome was serious infections, defined as requiring hospitalisation. Efficacy was assessed by serial HBI measurement and treatment persistence.

Results 70 patients were included, with a median age of 68 years (range 60-87), male:female ratio of 9:5 and median Charlson co-morbidity index of 4 (range 2-9). 44 (62.9\%) had prior anti-TNF exposure and 15 (21.4\%) previous vedolizumab. Median treatment duration was 12 months (range 2-

\begin{tabular}{lll} 
Abstract PM0-36 & Table 1 & infection aetiology and severity \\
\hline Severe & Aetiology & Number of patients (\%) \\
& Pneumonia & $3(4.3)$ \\
& Line infection & $2(2.9)$ \\
& Covid-19 & $1(1.4)$ \\
& Biliary & $1(1.4)$ \\
& Epididymo-orchitis & $1(1.4)$ \\
Non-severe & Shingles & $1(1.4)$ \\
& Lower respiratory tract & $9(12.9)$ \\
& Urinary & $6(8.6)$ \\
& Coryzal & $4(5.7)$ \\
& Pneumonia & $1(1.4)$ \\
& Diarrhoea & $1(1.4)$ \\
& Line infection & $1(1.4)$ \\
& Soft tissue & $1(1.4)$ \\
\hline
\end{tabular}

48), with a total of 84 patient years. 31 patients (41.3\%) had steroids at initiation and $33(47.1 \%)$ required a later course of steroids.

7 patients (10\%) had a combined 9 serious infections, of which 1 was life threatening requiring organ support. Incidence of serious infections was 0.107 per patient year. A further 18 had a combined 22 non-severe infections (Table 1). The overall infection rate was 0.42 per patient year. Charlson co-morbidity index was numerically higher in those developing severe infections (median 5, range 3-7 vs. median 4, range 29, $\mathrm{P}=\mathrm{NS}$ ). 3 patients developed a malignancy; non-Hodgkin's lymphoma, melanoma and pancreatic cancer.

Mean HBI improved from baseline 8.13 to 4.64 at 6 months and 4.10 at last follow up (both $\mathrm{P}<0.0001$ ). Treatment persistence rate was $61.4 \%(\mathrm{~N}=43)$ and $36(51.4 \%)$ were steroid-free. Reasons for discontinuation were primary non-response (42\%), adverse event (32\%), secondary loss of response (10\%), malignancy (10\%) and lack of funding (5\%). Conclusion Ustekinumab was safe and effective in a cohort of elderly CD patients. Infections were mostly mild and did not result in therapy discontinuation. Risk of serious infection was very low at 0.107 per patient year of treatment.

\section{PM0-37 COVID-19 IMPACT ON CARE AND PRESCRIBING FOR INFLAMMATORY BOWEL DISEASE: DATA FROM THE IBD REGISTRY}

${ }^{1}$ Keith Bodger*, ${ }^{2}$ Stuart Bloom, ${ }^{2}$ Liz Dobson, ${ }^{2}$ Fraser Cummings, ${ }^{2}$ Stephen Grainger, ${ }^{2}$ Nick Kennedy, on behalf of the UK IBD Registry Collaboration. ${ }^{1}$ University of Liverpool, Liverpool, UK; ${ }^{2} U K$ IBD Registry, London, UK

\subsection{6/gutjnl-2021-BSG.176}

Introduction The first wave of the COVID-19 pandemic saw a sharp rise in UK cases during March 2020. We analysed UK IBD Registry data to investigate changes in contacts and prescribing in the immediate post-COVID period to gain insights into the impact of the pandemic on IBD care.

Methods We aggregated quarterly data (Jan-Mar 2019 to AprJun 2020), extracting counts of clinical events (outpatient contacts and biologics reviews), contact types (face-to-face, 'F2F'; or telephone/virtual, 'non-F2F'), new diagnoses and drug starts (oral steroids, further categorised as prednisolone and non- 